\title{
Similarity of Hybrid Numbers
}

\author{
İskender Öztürk ${ }^{1}$ and MUSTAFA ÖZDEMIR ${ }^{2}$ \\ ${ }^{1}$ Akdeniz University \\ ${ }^{2}$ Akdeniz Universitesi
}

May 5, 2020

\begin{abstract}
The set of hybrid numbers is a noncommutative number system unified and generalized the complex, dual and double(hyperbolic) numbers with the relation $\mathbf{i h}=-\mathbf{h i}=\varepsilon+\mathbf{i}$. Two hybrid numbers $\mathbf{p}$ and $\mathbf{q}$ are said to be similar if there exist a hybrid number $\mathbf{x}$ satisfying the equality $\mathbf{x}^{-1} \mathbf{q} \mathbf{x}=\mathbf{p}$. And it is denoted by $p \sim q$. In this paper, we study the concept of similarity for hybrid numbers by solving the linear equations $\mathbf{p x}=\mathbf{x q}$ and $\mathbf{q x}-\mathbf{x p}=\mathbf{c}$ for $\mathbf{p}, \mathbf{q}, \mathbf{c} \in \mathbb{K}$.
\end{abstract}

Keywords : Hybrid numbers, quaternions, coquaternions, dual and double numbers.

MSC Classification : 15A66, 11R52, 15A18, 15A06, 16S50.

\section{Hosted file}

mma_hybrid.pdf available at https://authorea.com/users/295812/articles/424681-similarity-ofhybrid-numbers

\section{Introduction}

The concept of similarity is one of the important subjects in Linear algebra. Two square matrices $A$ and $B$ are called similar if $B=P^{-1} A P$ for some invertible square matrix $P$. Similar matrices have many common properties such as rank, determinant, trace, eigenvalues, characteristic polynomial etc. Two square matrices are similar if and only if they have the same Jordan normal form. There is the concept of similarity in number systems as in the similarity of matrices. It is known that two complex numbers are similar to each other if and only if they are equal. However, this definition is different for quaternions since quaternion algebra is non-commutative. Two quaternions (or coquaternions) $\mathbf{p}$ and $\mathbf{q}$ are said to be similar if there exists a quaternion $\boldsymbol{a} \neq 0$, satisfying the equality $\boldsymbol{a}^{-1} \mathbf{p} \boldsymbol{a}=\mathbf{q}$ (Zhang \& matrices of quaternions, n.d.), (Falcão et al., n.d.), (Falcão et al., n.d.). This definition equivalent to the linear equation $\mathbf{p x}=\mathbf{x q}$ has a nonzero solution $\mathbf{x}$. If $\mathbf{p}$ and $\mathbf{q}$ are similar, then it is denoted by $\mathbf{p} \sim \mathbf{q}$. Besides, Two quaternions $\mathbf{q}=S_{\mathbf{q}}+\mathbf{V}_{\mathbf{q}}$ and $\mathbf{p}=S_{\mathbf{p}}+\mathbf{V}_{\mathbf{p}}$ are similar if and only if $S_{\mathbf{q}}=S_{\mathbf{p}}$ and $\left\|\mathbf{V}_{\mathbf{q}}\right\|=\left\|\mathbf{V}_{\mathbf{p}}\right\|$. The similarity of quaternions is an equivalent relation. The roots of split quaternions can be examined with the help of some classifications, which is the result of similarity ?, (Özdemir, n.d.).

Besides, we encounter the concepts of semisimilarity and con-semisimilarity as well as the similarity of quaternions in the literature (Zhang \& matrices of quaternions, n.d.), (Liping \& of quaternion matrices and, n.d.). Two quaternions $\mathbf{p}$ and $\mathbf{q}$ are said to be semi-similar if there exist quaternions $\mathbf{x}$ and $\mathbf{y}$ satisfying equations $\mathbf{x p y}=\mathbf{q}$ and $\mathbf{y} \mathbf{p} \mathbf{x}=\mathbf{q}$. Also, $\mathbf{p}$ and $\mathbf{q}$ are said to be con-semisimilar if there are $\mathbf{x}$ and $\mathbf{y}$ satisfying the equalities $\overline{\mathbf{x}} \mathbf{p} \mathbf{y}=\mathbf{q}$ and $\overline{\mathbf{y}} \mathbf{q} \mathbf{x}=\mathbf{p}$ (Y. \& two pairs of quadratic equations in, n.d.), (Tian et al., n.d.). The semi-similarity was first defined and studied by Hartwing and Bevis (E. \& for matrices, n.d.), (Bevis et al., n.d.). Both semi-similarity and con-semi-similarity are equivalence relations. In this study, we will not deal with the concept of semi-similarity and con-semi-similarity. 
The similarity of quaternions and quaternion matrices is studied by many mathematicians. Similarity in quaternion matrices is not as clear as the similarity of real and complex matrices, since the quaternion product is non-commutative. So, it is not easy to solve linear and nonlinear equations in quaternions. Wolf proved that the quaternion matrices $A$ and $B$ are similar if and only if the real matrices $\chi(A)$ and $\chi(B)$ are similar, where the matrix

$$
\chi(A)=[
$$

$\frac{A_{1}}{-\overline{A_{2}}} \quad \frac{A_{2}}{A_{1}}$ is the adjoint of the quaternion matrix $A=A_{1}+A_{2} \mathbf{j}$ (Wolf $\&$ of matrices in which the elements are, n.d.), (Tian \& over, n.d.), (Zhang \& matrices of quaternions, n.d.). The similarity of quaternion matrices is closely related to the solution of linear quaternionic equations $\mathbf{a x}-\mathbf{x b}=\mathbf{c}, \mathbf{a x}-\mathbf{x} \overline{\mathbf{b}}=\mathbf{c}$ and $\mathbf{x a} \overline{\mathbf{x}}=\mathbf{b}$. Therefore, the solution of these linear equations in the set of quaternions and split quaternions has been studied by many mathematicians (M. \& linear split, n.d.), ?, (Y. \& two pairs of quadratic equations in, n.d.), (Kula et al., n.d.).

In this article, the definition and some properties of similarity of hybrid numbers are studied. The hybrid number system is a set of numbers defined by Özdemir in 2018 and combining dual, complex and perplex (hyperbolic) number sets. The set of hybrid numbers is isomorphic to split quaternions. Therefore, results similar to those in split quaternions will be obtained. In the first section, this number set is summarized briefly. Detailed information for hybrid numbers can be found in Özdemir's articles ?, (Özdemir \& n-th roots of a $2 \times 2$ real, n.d.).

\section{Hybrid Numbers}

The set of hybrid numbers, which is a noncommutative ring, is a generalization of complex, hyperbolic and dual number sets and it is defined as

$$
\mathbb{K}=\left\{a+b \mathbf{i}+c \varepsilon+d \mathbf{h}: a, b, c, d \in \mathbb{R}, \mathbf{i}^{2}=-1, \varepsilon^{2}=0, \mathbf{h}^{2}=1, \mathbf{i h}=-\mathbf{h i}=\varepsilon+\mathbf{i}\right\} .
$$

Multiplication table of hybrid numbers as follows.

\begin{tabular}{|l|l|l|l|}
\hline$\cdot$ & $\mathbf{i}$ & $\varepsilon$ & $\mathbf{h}$ \\
\hline $\mathbf{i}$ & $-\mathbf{1}$ & $\mathbf{1}-\mathbf{h}$ & $\varepsilon+\mathbf{i}$ \\
\hline$\varepsilon$ & $\mathbf{h}+\mathbf{1}$ & $\mathbf{0}$ & $-\varepsilon$ \\
\hline $\mathbf{h}$ & $-\varepsilon-\mathbf{i}$ & $\varepsilon$ & $\mathbf{1}$ \\
\hline
\end{tabular}

Hybrid numbers are classified as timelike, spacelike and lightlike such as in split quaternions. Since the algebra of hybrid numbers is isomorphic to the algebra of $2 \times 2$ real matrices, $2 \times 2$ matrices can be classified with respect to kind of corresponding hybrid number. So, hybrid numbers provide great simplicity for finding $n$-th roots of $2 \times 2$ matrices. We can define polar form of a $2 \times 2$ matrix and use De Moivre's formulas to find $n$-th roots of $2 \times 2$ matrices?.

Many definitions such as conjugate and norm in hybrid numbers are similar to those in split quaternions. The conjugate of a hybrid number $\mathbf{q}=a+b \mathbf{i}+c \varepsilon+d \mathbf{h}=\operatorname{Re} \mathbf{q}+\operatorname{Imq}$ is defined as $\overline{\mathbf{q}}=\operatorname{Req}-\operatorname{Imq}=a-b \mathbf{i}-c \varepsilon-d \mathbf{h}$. We say that a hybrid number; 
$\mathbf{q}$ is spacelike if $\mathcal{C}_{\mathbf{q}}<0$;

$\mathbf{q}$ is timelike if $\mathcal{C}_{\mathbf{q}}>0$; where $\mathcal{C}_{\mathbf{q}}=\mathbf{q} \overline{\mathbf{q}}=\overline{\mathbf{q}} \mathbf{q}=a^{2}+(b-c)^{2}-c^{2}-d^{2}$. The inverse of a hybrid number $\mathbf{q}$ is lightlike if $\mathcal{C}_{\mathbf{q}}=0$.

$\mathbf{q}=a+b \mathbf{i}+c \varepsilon+d \mathbf{h}$ is found as $\mathbf{q}^{-1}=\overline{\mathbf{q}} / \mathcal{C}_{\mathbf{q}}$.for $\|\mathbf{q}\| \neq 0$. Lightlike hybrid numbers have no inverse. Besides, norm of $\mathbf{q}$ is defined as

$$
\|\mathbf{q}\|=\sqrt{\left|\mathcal{C}_{\mathbf{q}}\right|}=\sqrt{\left|a^{2}+(b-c)^{2}-c^{2}-d^{2}\right|} .
$$

This norm definition is also consistent with the norm definition in complex, dual and double numbers. Algebraic and geometric properties of these three number system can be found in the articles (Kisil et al., n.d.), (A. et al., n.d.), (F. et al., n.d.), (J. et al., n.d.). The vector, $\mathcal{E}_{\mathbf{q}}=((b-c), c, d)$ is called the hybrid vector of q. We say that a hybrid number;

$\begin{array}{ll}\mathbf{q} \text { is elliptic } & \text { if } \triangle_{\mathbf{q}}<0 ; \\ \mathbf{q} \text { is hyperbolic } & \text { if } \triangle_{\mathbf{q}}>0 ; \text { where } \triangle_{\mathbf{q}}=-(b-c)^{2}+c^{2}+d^{2} \text {. Also, the real number } \sqrt{\left|\triangle_{\mathbf{q}}\right|} \text { will be called }\end{array}$ $\mathbf{q}$ is parabolic if $\triangle_{\mathbf{q}}=0$.

as the norm of the hybrid vector of $\mathbf{q}$ and will be denoted by $\left\|\mathcal{E}_{\mathbf{q}}\right\|$. For detailed information about hybrid numbers see the references (Özdemir \& Introduction to hybrid numbers, n.d.), (missing citation) and ?.

\section{Matrix of hybrid numbers}

Let $\mathbf{q}=q_{1}+q_{2} \mathbf{i}+q_{3} \varepsilon+q_{4} \mathbf{h}$ and $\mathbf{x}$ be two hybrid numbers. The linear transformations of the left and right multiplications in $\mathbb{K}$ are defined as,

$$
\begin{aligned}
& \varphi, \tau: \mathbb{K} \rightarrow \mathbb{K} \\
& \varphi(\mathbf{q})=\mathbf{q} \mathbf{x} \quad \text { and } \quad \tau(\mathbf{q})=\mathbf{x q}
\end{aligned}
$$

respectively. The matrix representations of these transformations are as follows:

$$
\varphi(\mathbf{q})=
$$

$$
\begin{aligned}
& {\left[\begin{array}{cccc}
q_{1} & q_{3}-q_{2} & q_{2} & q_{4} \\
q_{2} & q_{1}-q_{4} & 0 & q_{2} \\
q_{3} & -q_{4} & q_{1}+q_{4} & q_{2}-q_{3} \\
q_{4} & q_{3} & -q_{2} & q_{1}
\end{array}\right], \quad \tau(\mathbf{q})=} \\
& {\left[\begin{array}{cccc}
q_{1} & q_{3}-q_{2} & q_{2} & q_{4} \\
q_{2} & q_{1}+q_{4} & 0 & -q_{2} \\
q_{3} & q_{4} & q_{1}-q_{4} & q_{3}-q_{2} \\
q_{4} & -q_{3} & q_{2} & q_{1}
\end{array}\right] \quad(2)}
\end{aligned}
$$

Notice that, the eigenvalues of $\varphi(\mathbf{q})$ and $\tau(\mathbf{q})$ are $q_{1}+\left\|\mathcal{E}_{\mathbf{q}}\right\|, q_{1}-\left\|\mathcal{E}_{\mathbf{q}}\right\|$ and $q_{1}+\left\|\mathcal{E}_{\mathbf{q}}\right\|, q_{1}-\left\|\mathcal{E}_{\mathbf{q}}\right\|$ respectively. Also, we have

$$
\operatorname{det} \varphi(\mathbf{q})=\operatorname{det} \tau(\mathbf{q})=\|\mathbf{q}\|^{2} .
$$

It is clear that the eigenvalues of $\varphi(\mathbf{q})$ and $\tau(\mathbf{q})$ are equal and they can be given by the Table 1 , according to character and type of the hybrid number. 


\begin{tabular}{|c|c|c|c|}
\hline Type $\backslash$ Character & Spacelike & Lightlike & Timelike \\
\hline Hyperbolic & $q_{1} \pm\left\|\mathcal{E}_{\mathbf{q}}\right\|$ & $q_{1}$ & $q_{1} \pm\left\|\mathcal{E}_{\mathbf{q}}\right\|$ \\
\hline Parabolic & - & 0 & $q_{1}$ \\
\hline Elliptic & - & - & $q_{1} \pm\left\|\mathcal{E}_{\mathbf{q}}\right\| \mathbf{i}$ \\
\hline
\end{tabular}

Table 1 : Eigenvalues of the matrices $\varphi(q)$ and $\tau(q)$

Using the matrices $\varphi(\mathbf{q})$ and $\tau(\mathbf{q})$, it can be obtained following corollary.

Lemma 1. Let $\mathbf{p}, \mathbf{q} \in \mathbb{K}$ and $\lambda \in \mathbb{R}$. Then

1. $\varphi(\mathbf{q})=\varphi(\mathbf{p}) \Longleftrightarrow \mathbf{q}=\mathbf{p} \Longleftrightarrow \tau(\mathbf{q})=\tau(\mathbf{p})$

2. $\varphi(\mathbf{q}+\mathbf{p})=\varphi(\mathbf{q})+\varphi(\mathbf{p}), \quad \tau(\mathbf{q}+\mathbf{p})=\tau(\mathbf{q})+\tau(\mathbf{p})$

3. $\varphi(\mathbf{q p})=\varphi(\mathbf{q}) \varphi(\mathbf{p}), \quad \tau(\mathbf{q p})=\tau(\mathbf{q}) \tau(\mathbf{p})$

4. $\varphi(\mathbf{q}) \tau(\mathbf{p})=\tau(\mathbf{p}) \varphi(\mathbf{q})$

5. $\varphi(\lambda \mathbf{q})=\varphi(\mathbf{q} \lambda)=\lambda \varphi(\mathbf{q}), \quad \tau(\lambda \mathbf{q})=\tau(\mathbf{q} \lambda)=\lambda \tau(\mathbf{q})$

6. $\varphi(1)=\tau(1)=\mathbf{I}_{4}$

7. $\varphi(\mathbf{q})+\varphi(\overline{\mathbf{q}})=2 q_{1} \mathbf{I}_{4}, \quad \tau(\mathbf{q})+\tau(\overline{\mathbf{q}})=2 q_{1} \mathbf{I}_{4}$

8. $\varphi\left(\mathbf{q}^{-1}\right)=(\varphi)^{-1}(\mathbf{q}), \quad \tau\left(\mathbf{q}^{-1}\right)=\tau^{-1}(\mathbf{q}) \quad$ where $\quad\|\mathbf{q}\| \neq 0$

9. $\varphi(\mathbf{q}) a \varphi(\mathbf{q})=a, \quad \operatorname{det} \varphi(\mathbf{q})=1$

10. $\xi \tau(\overline{\mathbf{q}})=\varphi(\mathbf{q}) \xi, \quad \xi=[$

10

$\begin{array}{ll}0 & -\mathbf{I}_{3}\end{array}$

11. $\operatorname{det} \varphi(\mathbf{q})=\operatorname{det} \tau(\mathbf{q})=\|\mathbf{q}\|^{4}$

12. $\varphi\left(\mathbf{q}^{-1}\right)=\xi \tau_{\mathbb{K}}\left(\overline{\mathbf{q}^{-1}}\right) \xi=\{$
$\frac{1}{C^{2}(\mathbf{q})} \xi \tau(\overline{\mathbf{q}}) \xi$,
$\frac{-1}{C^{2}(\mathbf{q})} \xi \tau(\overline{\mathbf{q}}) \xi$,
$\mathbf{q}$ is spacelike
It has generalized inverse $\mathbf{q}$ is lightlike
Theorem 2. Let $\mathbf{p}, \mathbf{q} \in \mathbb{K}$. Then

$$
\begin{aligned}
\overrightarrow{\mathbf{q x}}=\varphi(\mathbf{q}) \overrightarrow{\mathbf{x}} & \\
\mathbf{x q}=\tau(\mathbf{q}) \overrightarrow{\mathbf{x}} & \overrightarrow{\mathbf{q} \mathbf{p}}=\varphi(\mathbf{q}) \tau(\mathbf{p}) \overrightarrow{\mathbf{x}}=\tau(\mathbf{p}) \varphi(\mathbf{q}) \vec{x}
\end{aligned}
$$

and

$$
\varphi(\mathbf{q}) \tau(\mathbf{p})=\tau(\mathbf{p}) \varphi(\mathbf{q}) .
$$


Proof. Let real vector representation of $\mathbf{q} \in \mathbb{K}$ be $\overrightarrow{\mathbf{q}}$. Then, it can be expressed as

$$
\overrightarrow{\mathbf{q}}=\varphi(\mathbf{q}) \mathbf{b}^{\mathrm{t}}, \quad \overrightarrow{\mathbf{q}}=\tau(\mathbf{q}) \mathbf{b}^{\mathrm{t}}, \quad \mathbf{b}=(1,0,0,0) .
$$

According to Corollary 1, we have

$$
\begin{aligned}
& \overrightarrow{\mathbf{q}}=\varphi(\mathbf{q x}) \mathbf{b}^{\mathrm{t}}=\varphi(\mathbf{q}) \varphi(\mathbf{x}) \mathbf{b}^{\mathrm{t}}=\varphi(\mathbf{q}) \overrightarrow{\mathbf{x}} \\
& \overrightarrow{\mathbf{x} \mathbf{q}}=\tau(\mathbf{x q}) \mathbf{b}^{\mathrm{t}}=\tau(\mathbf{q}) \tau(\mathbf{x}) \mathbf{b}^{\mathrm{t}}=\tau(\mathbf{q}) \overrightarrow{\mathbf{x}}
\end{aligned}
$$

and

$$
\begin{aligned}
& \overrightarrow{\mathbf{q x p}}=\overrightarrow{\mathbf{q}(x \mathbf{p})}=\varphi(\mathbf{q}) \overrightarrow{(x \mathbf{p})}=\varphi(\mathbf{q}) \tau(\mathbf{p}) \overrightarrow{\mathbf{x}} \\
& \overrightarrow{\mathbf{q x p}}=\overrightarrow{(\mathbf{q} x) \mathbf{p}}=\tau(\mathbf{p}) \overrightarrow{(x \mathbf{p})}=\tau(\mathbf{p}) \varphi(\mathbf{q}) \overrightarrow{\mathbf{x}}
\end{aligned}
$$

Since these equations are provided that for all $\mathbf{p}, \mathbf{q} \in \mathbb{K}$, they can be written,

$$
\varphi(\mathbf{q}) \tau(\mathbf{p})=\tau(\mathbf{p}) \varphi(\mathbf{q})
$$

\section{Generalized inverse}

In the next chapters, we will need the generalized inverse of a matrix to solve some systems of linear equations. Let's give this definition and some features. For detalied infomation see (Ben-Israel \& inverses: n.d.) and (missing citation).

Definition 3. Let $\mathbf{A}$ be an $m \times n$ matrix. The $n \times m$ matrix $\mathbf{G}$, which provides the equation $\mathbf{A G A}=\mathbf{A}$, is called the generalized inverse of the matrix $\mathbf{A}$.

Theorem 4. Let

$$
\mathbf{A}=
$$

$$
\begin{aligned}
& {\left[\begin{array}{ll}
\mathbf{R} & \mathrm{B} \\
\mathrm{C} & \mathrm{D}
\end{array}\right]=} \\
& {\left[\begin{array}{cc}
\mathbf{R} & \mathrm{B} \\
\mathrm{C} & \mathrm{CR}^{-1} \mathrm{~B}
\end{array}\right]}
\end{aligned}
$$

be an $m \times n$ matrix with $r=\operatorname{rank}(\mathbf{A})$, where $\mathbf{R}$ is an invertible $r \times r$ matrix. Then, the generalized inverse of $\mathbf{A}$ is

$$
\mathbf{G}=
$$

$\left[\begin{array}{ll}\mathbf{R}^{-1} & {[\mathbf{0}]_{1}} \\ {[\mathbf{0}]_{2}} & {[\mathbf{0}]_{3}}\end{array}\right]$

where $[\mathbf{0}]_{i}$ are represented zero matrices of dimension necessary to make $\mathbf{G}$ an $n \times m$ matrix. 
Theorem 5. Let $\mathbf{A}$ be an $m \times n$ matrix and its generalized inverse is $\mathbf{G}$. Then, for any fixed $\mathbf{y} \in \mathbb{R}^{m}$, the followings are satisfied.

1. $\mathbf{A x}=\mathbf{y}$, has a solution $\mathbf{x} \in \mathbb{R}^{n}$ if and only if $\mathbf{A G y}=\mathbf{y}$.

2. If the equation $\mathbf{A} \mathbf{x}=\mathbf{y}$ has a solution, then $\mathbf{x}$ is a solution of $\mathbf{A} \mathbf{x}=\mathbf{y}$ if and only if

$$
\mathbf{x}=\mathbf{G y}+(\mathbf{I}-\mathbf{G A}) \mathbf{z}
$$

for some $\mathbf{z} \in \mathbb{R}^{n}$

\section{The Similarity of Hybrid Numbers}

Definition 6. Let $\mathbf{q}$ and $\mathbf{p}$ be two hybrid numbers. $\mathbf{q}$ and $\mathbf{p}$ are called to be similar hybrid numbers, if there exists a hybrid number $\boldsymbol{a} \neq 0$, satisfying the equality $\boldsymbol{a}^{-1} \mathbf{q} \boldsymbol{a}=\mathbf{p}$, and it is denoted by $\mathbf{q} \sim \mathbf{p}$. The similarity relation " $\sim$ " on hybrid numbers is an equivalence relation, since it is reflexive, symmetric and transitive. It is clear that $\mathbf{q} \sim \mathbf{p}$ implies $\|\mathbf{q}\|=\|\mathbf{p}\|$, since $\left\|\boldsymbol{a}^{-1} \mathbf{q} \boldsymbol{a}\right\|=\|\mathbf{q}\|$.

Theorem 7. Let $\mathbf{p}, \mathbf{q} \in \mathbb{K}$. Then, $\mathbf{q} \sim \mathbf{p}$ if and only if

$$
\operatorname{Re}(\mathbf{q})=\operatorname{Re}(\mathbf{p}) \quad \text { and } \quad\left\|\mathcal{E}_{\mathbf{p}}\right\|=\left\|\mathcal{E}_{\mathbf{q}}\right\|
$$

Proof. Let $\mathbf{p}$ and $\mathbf{q}$ be similar hybrid numbers. From the corollary 1, we find

$$
\begin{gathered}
\operatorname{Re}(\mathbf{p})=\operatorname{Re}\left(\boldsymbol{a}^{-1} \mathbf{q} \boldsymbol{a}\right)=\operatorname{Re}\left(\boldsymbol{a}^{-1} \boldsymbol{a} \mathbf{q}\right)=\operatorname{Re}(\mathbf{q}) \\
\|\mathbf{p}\|=\left\|\boldsymbol{a}^{-1} \mathbf{q} \boldsymbol{a}\right\|=\left\|\boldsymbol{a}^{-1}\right\|\|\mathbf{q}\|\|\boldsymbol{a}\|=\|\mathbf{q}\|
\end{gathered}
$$

where $\boldsymbol{a}$ is a hybrid number and $\|\boldsymbol{a}\| \neq 0$. Considering the above equations, the equality $\left\|\mathcal{E}_{\mathbf{p}}\right\|=\left\|\mathcal{E}_{\mathbf{q}}\right\|$ is obtained.

Theorem 8. Let $\mathbf{q}=q_{1}+q_{2} \mathbf{i}+q_{3} \varepsilon+q_{4} \mathbf{h}$ and $\mathbf{p}=p_{1}+p_{2} \mathbf{i}+p_{3} \varepsilon+p_{4} \mathbf{h}$ be two hybrid numbers. If we denote as

$$
\Omega_{\mathbf{q p}}:=\varphi(\mathbf{q})-\tau(\mathbf{p}),
$$

then we have the following properties.

1. The determinant of the matrix $\Omega_{\mathbf{q p}}:=\varphi(\mathbf{q})-\tau(\mathbf{p})$ is,

$$
\operatorname{det} \Omega_{\mathbf{q p}}=\mathrm{s}^{4}-2 \mathrm{~s}^{2}\left(\triangle_{\mathbf{q}}+\triangle_{\mathbf{p}}\right)+\left(\triangle_{\mathbf{q}}-\triangle_{\mathbf{p}}\right)^{2},
$$

where $\mathrm{s}=q_{1}-p_{1}$. So, $\operatorname{det} \Omega_{\mathbf{q p}}=0$ if and only if $\operatorname{Re}(\mathbf{q})=\operatorname{Re}(\mathbf{p})$ and $\left\|\mathcal{E}_{\mathbf{q}}\right\|=\left\|\mathcal{E}_{\mathbf{p}}\right\|$.

2. The eigenvalues of the matrix $\Omega_{\mathbf{q p}}$ can be given in the following table. 


\begin{tabular}{|c|c|c|c|}
\hline$p \backslash q$ & Hyperbolic & Parabolic & Elliptic \\
\hline Hyperbolic & $\begin{array}{c}\mathrm{s}+\left|\triangle_{\mathbf{p}} \pm \triangle_{\mathbf{q}}\right| \\
\mathrm{s}-\left|\triangle_{\mathbf{p}} \pm \triangle_{\mathbf{q}}\right|\end{array}$ & $\mathrm{s} \pm \triangle_{\mathbf{p}}$ & $\begin{array}{c}\mathrm{s}+\left(\triangle_{\mathbf{p}} \pm \mathbf{i} \triangle_{\mathbf{q}}\right) \\
\mathrm{s}-\left(\triangle_{\mathbf{p}} \pm \mathbf{i} \triangle_{\mathbf{q}}\right)\end{array}$ \\
\hline Parabolic & $s \pm \triangle_{q}$ & $\mathrm{~S}$ & $\mathrm{~s} \pm \mathbf{i} \triangle_{\mathrm{q}}$ \\
\hline Elliptic & $\begin{array}{c}\mathrm{s}+\left(\mathbf{i} \triangle_{\mathbf{p}} \pm \triangle_{\mathbf{q}}\right) \\
\mathrm{s}-\left(\mathbf{i} \triangle_{\mathbf{p}} \pm \triangle_{\mathbf{q}}\right)\end{array}$ & $\mathrm{s} \pm \mathbf{i} \triangle_{\mathbf{p}}$ & $\begin{array}{l}s+\left(\triangle_{\mathbf{p}} \pm \triangle_{\mathbf{q}}\right) \mathbf{i} \\
\mathrm{s}-\left(\triangle_{\mathbf{p}} \pm \triangle_{\mathbf{q}}\right) \mathbf{i}\end{array}$ \\
\hline
\end{tabular}

Table 2: Eigenvalues of the matrix $\Omega_{\mathbf{q p}}$

3. If $q_{1} \neq p_{1}$ or $\left\|\mathcal{E}_{\mathbf{q}}\right\| \neq\left\|\mathcal{E}_{\mathbf{p}}\right\|$, then $\Omega_{\mathbf{q p}}$ is a nonsingular matrix and its inverse can be expressed as

$$
\begin{aligned}
\Omega_{\mathbf{q p}}^{-1} & =\varphi^{-1}\left(\mathbf{q}^{2}-2 p_{1} \mathbf{q}+\|\mathbf{p}\|^{2}\right)[\varphi(\mathbf{q})-\tau(\overline{\mathbf{p}})] \\
& =\varphi^{-1}\left(2 \mathbf{s q}+\|\mathbf{p}\|^{2}-\|\mathbf{q}\|^{2}\right)[\varphi(\mathbf{q})-\tau(\overline{\mathbf{p}})]
\end{aligned}
$$

or

$$
\begin{aligned}
\Omega_{\mathbf{q p}}^{-1} & =\tau^{-1}\left(\mathbf{p}^{2}-2 q_{1} \mathbf{p}+\|\mathbf{q}\|^{2}\right)[\varphi(\overline{\mathbf{q}})-\tau(\mathbf{p})] \\
& =\tau^{-1}\left(2 \mathbf{s} \mathbf{p}+\|\mathbf{q}\|^{2}-\|\mathbf{p}\|^{2}\right)[\varphi(\overline{\mathbf{q}})-\tau(\mathbf{p})]
\end{aligned}
$$

4. If $\mathbf{p}$ and $\mathbf{q}$ are non-parabolic similar hybrid numbers, then $\Omega_{\mathbf{q p}}$ is a singular matrix and its generalized inverse is

$$
\Omega_{\mathbf{q p}}^{-1}=\frac{1}{4\left|\triangle_{\mathbf{q}}\right|} \Omega_{\mathbf{q p}}
$$

5. If $\mathbf{p}$ and $\mathbf{q}$ are parabolic similar hybrid numbers with $\left\|\mathcal{E}_{\mathbf{q}}\right\|=\left\|\mathcal{E}_{\mathbf{p}}\right\|=0$, then the matrix $\Omega_{\mathbf{q p}}$ is also singular, and its generalized inverse is

$$
\Omega_{\mathbf{q p}}^{-1}=[
$$

$\begin{array}{cc}\mathrm{A}^{-1} & {[\mathbf{0}]} \\ {[\mathbf{0}]} & {[\mathbf{0}]}\end{array}$.where $\mathrm{A}=[$

$\begin{array}{cc}0 & p_{2}-p_{3}-q_{2}+q_{3} \\ q_{2}-p_{2} & -p_{4}-q_{4}\end{array}, q_{2} \neq p_{2}, p_{2}-p_{3} \neq q_{2}-q_{3}$ and, [0] is a 2 by 2 zero matrix. Besides, row echelon form of $\Omega_{\mathbf{q p}}$ is

$\begin{array}{cccc}1 & 0 & \frac{p_{4}+q_{4}}{p_{2}-p_{3}-q_{2}+q_{3}} & -\frac{p_{3}+q_{3}}{p_{2}-p_{3}-q_{2}+q_{3}} \\ 0 & 1 & \frac{q_{2}-p_{2}}{p_{2}-p_{3}-q_{2}+q_{3}} & \frac{q_{4}-p_{4}}{p_{2}-p_{3}-q_{2}+q_{3}}\end{array}$.

Proof. 1. Let $\mathbf{q}=q_{1}+q_{2} \mathbf{i}+q_{3} \varepsilon+q_{4} \mathbf{h}$ and $\mathbf{p}=p_{1}+p_{2} \mathbf{i}+p_{3} \varepsilon+p_{4} \mathbf{h} \in \mathbb{K}$ be two hybrid numbers, then from the equality $\Omega_{\mathbf{q p}}=\varphi(\mathbf{q})-\tau(\mathbf{p})$, we obtain

$$
\Omega_{\mathbf{q p}}=
$$




$$
\left[\begin{array}{cccc}
\mathrm{s} & p_{2}-p_{3}-q_{2}+q_{3} & q_{2}-p_{2} & q_{4}-p_{4} \\
q_{2}-p_{2} & \mathrm{~s}-p_{4}-q_{4} & 0 & p_{2}+q_{2} \\
q_{3}-p_{3} & -p_{4}-q_{4} & \mathrm{~s}+p_{4}+q_{4} & p_{2}-p_{3}+q_{2}-q_{3} \\
q_{4}-p_{4} & p_{3}+q_{3} & -p_{2}-q_{2} & \mathrm{~s}
\end{array}\right]
$$

where $\mathrm{s}=q_{1}-p_{1}$. Now, if we calculate determinant of $\Omega_{\mathbf{q p}}$, we obtain

$$
\begin{aligned}
\operatorname{det} \Omega_{\mathbf{q p}}= & \mathrm{s}^{4}+2 \mathrm{~s}^{2}\left(p_{2}^{2}-2 p_{2} p_{3}-p_{4}^{2}+q_{2}^{2}-2 q_{2} q_{3}-q_{4}^{2}\right) \\
& +\left(p_{2}^{2}-2 p_{2} p_{3}-p_{4}^{2}-q_{2}^{2}+2 q_{2} q_{3}+q_{4}^{2}\right)^{2} \\
= & \mathrm{s}^{4}-2 \mathrm{~s}^{2}\left(\triangle_{\mathbf{q}}+\triangle_{\mathbf{p}}\right)+\left(\triangle_{\mathbf{q}}-\triangle_{\mathbf{p}}\right)^{2}
\end{aligned}
$$

2. The characteristic polynomial of $\Omega_{\mathbf{q p}}$ is

$$
\left|\lambda \mathbf{I}-\Omega_{\mathbf{q p}}\right|=\left[(\lambda-\mathrm{s})^{2}-\left(\triangle_{\mathbf{q}}-\triangle_{\mathbf{p}}\right)^{2}\right]\left[(\lambda-\mathrm{s})^{2}-\left(\triangle_{\mathbf{q}}+\triangle_{\mathbf{p}}\right)^{2}\right] .
$$

According to this equality, we can see that accuracy of Table 2.

3. If we expand the product $[\varphi(\mathbf{q})-\tau(\overline{\mathbf{p}})] \Omega_{\mathbf{q p}}$, we obtain

$$
\begin{aligned}
& =\varphi\left(\mathbf{q}^{2}\right)-\varphi(\mathbf{q}) \tau(\mathbf{p})-\tau(\overline{\mathbf{p}}) \varphi(\mathbf{q})+\tau(\overline{\mathbf{p}}) \tau(\mathbf{p}) \\
& =\varphi\left(\mathbf{q}^{2}\right)-\varphi(\mathbf{q}) \tau(\mathbf{p})-\varphi(\mathbf{q}) \tau(\overline{\mathbf{p}})+\tau(\overline{\mathbf{p}} \mathbf{p}) \\
& =\varphi\left(\mathbf{q}^{2}\right)-\varphi(\mathbf{q}) \tau(\mathbf{p}+\overline{\mathbf{p}})+\tau(\overline{\mathbf{p}} \mathbf{p}) \\
& =\varphi\left(\mathbf{q}^{2}\right)-\varphi(\mathbf{q}) 2 p_{1}+\|\mathbf{p}\|^{2} \mathbf{I}_{4} \\
& =\varphi\left(\mathbf{q}^{2}-2 p_{1} \mathbf{q}+\|\mathbf{p}\|^{2}\right)
\end{aligned}
$$

and similarly, we can find the equality

$$
[\varphi(\overline{\mathbf{q}})-\tau(\mathbf{p})] \Omega_{\mathbf{q p}}=\tau\left(\mathbf{p}^{2}-2 q_{1} \mathbf{p}+\|\mathbf{q}\|^{2}\right) .
$$

If we multiply the first equation from left by $\varphi^{-1}\left(\mathbf{q}^{2}-2 p_{1} \mathbf{q}+\|\mathbf{p}\|^{2}\right)$ and right by $\Omega_{\mathbf{q p}}^{-1}$, then we obtain

$$
\begin{aligned}
\Omega_{\mathbf{q p}}^{-1} & =\varphi^{-1}\left(\mathbf{q}^{2}-2 p_{1} \mathbf{q}+\|\mathbf{p}\|^{2}\right)[\varphi(\mathbf{q})-\tau(\overline{\mathbf{p}})] \\
& =\varphi^{-1}\left(2 \mathrm{sq}-\|\mathbf{q}\|^{2}+\|\mathbf{p}\|^{2}\right)[\varphi(\mathbf{q})-\tau(\overline{\mathbf{p}})]
\end{aligned}
$$

Using the same way, we can calculate the below equation.

$$
\Omega_{\mathbf{q p}}^{-1}=\tau^{-1}\left(2 \mathbf{s p}+\|\mathbf{q}\|^{2}-\|\mathbf{p}\|^{2}\right)[\varphi(\overline{\mathbf{q}})-\tau(\mathbf{p})] .
$$

4. If $\mathbf{q}$ and $\mathbf{p}$ are similar non-parabolic hybrid numbers, then we have

$$
\Omega_{\mathbf{q p}}=\varphi(\mathbf{q})-\tau(\mathbf{p})=\varphi(\operatorname{Imq})-\tau(\operatorname{Imp}),
$$

and

$$
(\operatorname{Imq})^{2}=(\operatorname{Imp})^{2}=\left|\triangle_{\mathbf{q}}\right| .
$$

Now, let's calculate the below equation, 


$$
\begin{aligned}
\Omega_{\mathbf{q p}}^{3}= & {[\varphi(\operatorname{Imq})-\tau(\operatorname{Imp})]^{3} } \\
= & {[\varphi(\operatorname{Imq})]^{3}-3[\varphi(\operatorname{Imq})]^{2} \tau(\operatorname{Imp}) } \\
& +3 \varphi(\operatorname{Imq})[\tau(\operatorname{Imp})]^{2}-[\tau(\operatorname{Imp})]^{3} \\
= & \left|\triangle_{\mathbf{q}}\right| \varphi(\operatorname{Imq})-3[\varphi(\operatorname{Imq})]^{2} \tau(\operatorname{Imp}) \\
& +3\left|\triangle_{\mathbf{q}}\right| \varphi(\operatorname{Imq})-\left|\triangle_{\mathbf{q}}\right| \tau(\operatorname{Imp}) \\
= & 4\left|\triangle_{\mathbf{q}}\right|[\varphi(\operatorname{Imq})-\tau(\operatorname{Im} \mathbf{p})]
\end{aligned}
$$

So we obtain that, $\Omega_{\mathbf{q p}}^{3}=4\left|\triangle_{\mathbf{q}}\right| \Omega_{\mathbf{q p}}$ and consequently, we can find the equation.

$$
\Omega_{\mathbf{q p}}^{-1}=\frac{1}{4\left|\triangle_{\mathbf{q}}\right|} \Omega_{\mathbf{q p}}
$$

5. If $\mathbf{q}, \mathbf{p}$ are the parabolic hybrid numbers with $\left\|\mathcal{E}_{\mathbf{q}}\right\|=\left\|\mathcal{E}_{\mathbf{p}}\right\|=0$, row echelon form of the matrix $\Omega_{\mathbf{q p}}$ can be easily calculated. When the condition $C \mathbf{R}^{-1} B=D$ is true for $\Omega_{\mathbf{q p}}$, then according to Theorem 4, the generalized inverse of $\Omega_{\mathbf{q p}}$ is

$$
\Omega_{\mathbf{q p}}^{-1}=[
$$

$\begin{array}{cc}\mathbf{R}^{-1} & {[\mathbf{0}]} \\ {[\mathbf{0}]} & {[\mathbf{0}]}\end{array}$ and the condition $\Omega_{\mathbf{q p}} \Omega_{\mathbf{q p}}^{-1} \Omega_{\mathbf{q p}}=\Omega_{\mathbf{q p}}$ is provided.

Theorem 9. If $\mathbf{q}$ is a non-parabolic hybrid number and $\mathbf{q} \notin \mathbb{R}$. The general solution of the linear equation $\mathbf{q} \mathbf{x}=\mathbf{x q}$ is

$$
\mathbf{x}=\mathbf{z}+\frac{1}{\left|\triangle_{\mathbf{q}}\right|}(\operatorname{Im} \mathbf{q}) \mathbf{z}(\operatorname{Im} \mathbf{q})
$$

where $\mathbf{z}=z_{1}+z_{2} \mathbf{i}+z_{3} \varepsilon+z_{4} \mathbf{h}$ is an arbitrary hybrid number. If $\mathbf{q}$ is a parabolic hybrid number and the coefficients of its $\mathbf{i}$ and $\varepsilon$ components are non-zero, then the matrix $\Omega_{\mathbf{q p}}$ and its row echelon form are

$$
\begin{array}{ccccc}
0 & 2 q_{3} & -2 q_{2} & 0 \\
2 q_{2} & -2 q_{4} & 0 & 0 \\
2 q_{2}-2 q_{3} & -2 q_{4} & 2 q_{4} & 0 \\
& 0 & 0 & 0 & 0 \\
1 & 0 & -\frac{q_{4}}{q_{3}} & 0 & \\
0 & 1 & -\frac{q_{2}}{q_{3}} & 0 & \\
0 & 0 & 0 & 0 & \text { respectively. So, the general solution of the equation } \mathbf{q x}=\mathbf{x q} \text { is } \\
0 & 0 & 0 & 0
\end{array}
$$

$$
\mathbf{x}=z_{3} \frac{q_{4}}{q_{3}}+z_{3} \frac{q_{2}}{q_{3}} \mathbf{i}+z_{3} \varepsilon+z_{4} \mathbf{h}
$$

where $\mathbf{z}=z_{1}+z_{2} \mathbf{i}+z_{3} \varepsilon+z_{4} \mathbf{h} \in \mathbb{K}$ is an arbitrary hybrid number and $q_{3} \neq 0$.

Proof. Let $\mathbf{q}$ be a non-parabolic hybrid number. Then, according to the equation $\mathbf{q} \mathbf{x}=\mathbf{x q}$, we obtain

$$
[\varphi(\operatorname{Imq})-\tau(\operatorname{Imq})] \overrightarrow{\mathbf{x}}=\overrightarrow{0}
$$


The general solution of this equation can be expressed as

$$
\overrightarrow{\mathbf{x}}=2\left[\mathbf{I}_{4}-\Omega_{\mathbf{q p}}^{-1} \Omega_{\mathbf{q p}}\right] \overrightarrow{\mathbf{z}},
$$

where $\overrightarrow{\mathbf{z}}$ is an arbitrary real vector. If we use it in the Theorem $8-4$, we get

$$
\begin{aligned}
\overrightarrow{\mathbf{x}} & =2\left[\mathbf{I}_{4}-\frac{1}{4\left|\triangle_{\mathbf{q}}\right|} \Omega_{\mathbf{q q}}^{2}\right] \overrightarrow{\mathbf{z}} \\
& =2\left[\mathbf{I}_{4}-\frac{1}{4\left|\triangle_{\mathbf{q}}\right|}\left(2\left|\triangle_{\mathbf{q}}\right| \mathbf{I}_{4}-2 \varphi_{\mathbb{K}}(\operatorname{Imq}) \tau(\operatorname{Imq})\right)\right] \overrightarrow{\mathbf{z}} \\
& =\left[\mathbf{I}_{4}+\frac{1}{\left|\triangle_{\mathbf{q}}\right|} \varphi(\operatorname{Imq}) \tau(\operatorname{Imq})\right] \overrightarrow{\mathbf{z}} \\
& =\mathbf{z}+\frac{1}{\left|\triangle_{\mathbf{q}}\right|}(\operatorname{Im} \mathbf{q}) \mathbf{z}(\operatorname{Imq}) .
\end{aligned}
$$

Therefore, if we take $\mathbf{z}=(\operatorname{Imq}) z_{0}$ in, (4), we find that

$$
\begin{aligned}
\mathbf{x} & =(\operatorname{Imq}) z_{0}+\frac{1}{\left|\triangle_{\mathbf{q}}\right|}(\operatorname{Imq})(\operatorname{Imq}) z_{0}(\operatorname{Imq}) \\
& =(\operatorname{Imq}) z_{0}+z_{0}(\operatorname{Imq}) \\
& =(\operatorname{Imq})\left(\operatorname{Re} z_{0}+\operatorname{Im} z_{0}\right)+\left(\operatorname{Re} z_{0}+\operatorname{Im} z_{0}\right)(\operatorname{Imq} \mathbf{q}) \\
& =2 \operatorname{Re} z_{0}(\operatorname{Imq})+(\operatorname{Imq})\left(\operatorname{Im} z_{0}\right)+\left(\operatorname{Im} z_{0}\right)(\operatorname{Imq}) .
\end{aligned}
$$

So, we can see that

$$
(\operatorname{Imq})\left(\operatorname{Im} z_{0}\right)+\left(\operatorname{Im} z_{0}\right)(\operatorname{Imq}) \in \mathbb{R}
$$

If $\mathbf{q}$ is a parabolic hybrid number and $\mathbf{x}=x_{1}+x_{2} \mathbf{i}+x_{3} \varepsilon+x_{4} \mathbf{h}$, then, we obtain the matrices (??). The equation $\mathbf{q x}=\mathbf{x q}$ is identical to $\Omega_{\mathbf{q q}} \mathbf{x}=0$ and it can be solved by writing this equation

$\left.\begin{array}{cccc}0 & 2 q_{3} & -2 q_{2} & 0 \\ 2 q_{2} & -2 q_{4} & 0 & 0 \\ 2 q_{2}-2 q_{3} & -2 q_{4} & 2 q_{4} & 0 \\ 0 & 0 & 0 & 0\end{array}\right]$

$z_{1}$

$\begin{aligned} & z_{2} \\ & z_{3}\end{aligned}=0$.According to Theorem 5 , a solution of the equation $\mathbf{q} \mathbf{x}=\mathbf{x q}$ is

$z_{4}$

$$
\mathbf{x}=[
$$

$\left.\begin{array}{cccc}\frac{q_{4}}{2 q_{2} q_{3}} & \frac{1}{2 q_{2}} & 0 & 0 \\ \frac{1}{2 q_{3}} & 0 & 0 & 0 \\ 0 & 0 & 0 & 0 \\ 0 & 0 & 0 & 0\end{array}\right]$




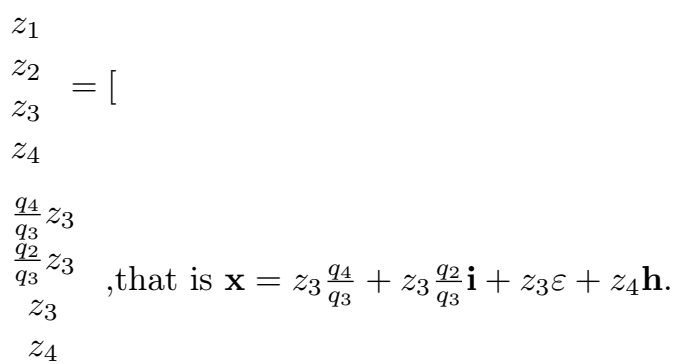

Corollary 10. If $\mathbf{q}$ is a non-parabolic hybrid number and $\mathbf{q} \notin \mathbb{R}$, then the general solution of the linear equation $\mathbf{q} \mathbf{x}=\mathbf{x q}$ is $\mathbf{x}=\lambda_{0}+\lambda_{1} \mathbf{q}$, where $\lambda_{0}=(\operatorname{Imq})\left(\operatorname{Im} z_{0}\right)+\left(\operatorname{Im} z_{0}\right)(\operatorname{Imq})$ and $\lambda_{1}=2 \operatorname{Re}\left(z_{0}\right)$.

Example 11. Let's find the solution of the equation $\mathbf{q x}=\mathbf{x q}$ for the hybrid number $\mathbf{q}=q_{1}+q_{2} \mathbf{i}+q_{3} \varepsilon+q_{4} \mathbf{h}$. The general solution is $\mathbf{x}=\lambda_{0}+\lambda_{1} \mathbf{q}$, where

$$
\lambda_{0}=(\operatorname{Imq})\left(\operatorname{Im} z_{0}\right)+\left(\operatorname{Im} z_{0}\right)(\operatorname{Imq})=
$$

$\left[2 c q_{2}+2 d q_{4}-2 b\left(q_{2}-q_{3}\right) \quad 0 \quad 0 \quad 0\right]{ }^{\mathrm{t}}$ and, $\lambda_{1}=2 \operatorname{Re}\left(z_{0}\right)=2 a$ and, $\mathbf{z}=a+b \mathbf{i}+c \varepsilon+d \mathbf{h}$ is arbitrary hybrid number. Therefore, the general solution of $\mathbf{q} \mathbf{x}=\mathbf{x q}$ is

$$
x=\lambda_{0}+\lambda_{1} \mathbf{q}=[
$$

$2 a q_{1}+2 c q_{2}+2 d q_{4}-2 b\left(q_{2}-q_{3}\right) \quad 2 a q_{2} \quad 2 a q_{3} \quad 2 a q_{4}{ }^{\mathrm{t}}$. Actually, one can see the accuracy of this solution by checking in the equation.

Theorem 12. Let $\mathbf{q}, \mathbf{p} \in \mathbb{K}$ be two non-parabolic hybrid numbers. Then we have the following properties. 1. The linear equation $\mathbf{q} \mathbf{x}=\mathbf{x p}$ has a nonzero solution if and only if $\operatorname{Re}(\mathbf{q})=\operatorname{Re}(\mathbf{p})$, and $\left\|\mathcal{E}_{\mathbf{q}}\right\|=\left\|\mathcal{E}_{\mathbf{p}}\right\|$. 2. In that case, the general solution of the equation $\mathbf{q} \mathbf{x}=\mathbf{x p}$ is

$$
\mathbf{x}=\mathbf{z}+\frac{1}{\left\|\mathcal{E}_{\mathbf{q}}\right\|\left\|\mathcal{E}_{\mathbf{p}}\right\|}(\operatorname{Imq}) \mathbf{z}(\operatorname{Imp})
$$

where $\mathbf{z} \in \mathbb{K}$ is an arbitrary hybrid number. In particular, if $\mathbf{p} \neq \overline{\mathbf{q}}$ and Imq + Im $\mathbf{p} \neq 0$, then the general solution of $\mathbf{q} \mathbf{x}=\mathbf{p} \mathbf{x}$ can be written as

$$
\mathbf{x}=\lambda_{0}(\operatorname{Imq}+\operatorname{Imp})+\lambda_{1}\left[\left\|\mathcal{E}_{\mathbf{q}}\right\|\left\|\mathcal{E}_{\mathbf{p}}\right\|+(\operatorname{Im} \mathbf{q})(\operatorname{Imp})\right]
$$

where $\lambda_{0}, \lambda_{1}$ are arbitrary real numbers.

Proof. According to Theorem 8 , the equation $\mathbf{q} \mathbf{x}=\mathbf{p x}$ is equivalent to

$$
[\varphi(\mathbf{q})-\tau(\mathbf{p})] \overrightarrow{\mathbf{x}}=\Omega_{\mathbf{q p}} \overrightarrow{\mathbf{x}}=0
$$

And this equation has a nonzero solution if and only if $\left|\Omega_{\mathbf{q p}}\right|=0$, which is equivalent to Req $=$ Rep, and $\left\|\mathcal{E}_{\mathbf{q}}\right\|=\left\|\mathcal{E}_{\mathbf{p}}\right\|$, according to Theorem 8-4. So, the general solution of this equation can be written as 


$$
\overrightarrow{\mathbf{x}}=2\left[\mathbf{I}_{4}-\Omega_{\mathbf{q p}}^{-1} \Omega_{\mathbf{q p}}\right] \overrightarrow{\mathbf{z}}
$$

where $\overrightarrow{\mathbf{z}}$ is an arbitrary real vector. Then, using $\Omega_{\mathbf{q p}}^{-1}$ in Theorem $8-4$, we find

$$
\begin{aligned}
\overrightarrow{\mathbf{x}} & =2\left[\mathbf{I}_{4}-\Omega_{\mathbf{q p}}^{-1} \Omega_{\mathbf{q p}}\right] \overrightarrow{\mathbf{z}} \\
& =2\left[\mathbf{I}_{4}-\frac{1}{4\left|\triangle_{\mathbf{q}}\right|} \Omega^{2}(\mathbf{q}, \mathbf{p})\right] \overrightarrow{\mathbf{z}} \\
& =2\left[\mathbf{I}_{4}-\frac{1}{4\left|\triangle_{\mathbf{q}}\right|}\left(2\left|\triangle_{\mathbf{q}}\right| \mathbf{I}_{4}-2 \varphi(\operatorname{Imq}) \tau(\operatorname{Imp})\right)\right] \overrightarrow{\mathbf{z}} \\
& =\left[\mathbf{I}_{4}+\frac{1}{\left|\triangle_{\mathbf{q}}\right|} \varphi(\operatorname{Imq}) \tau(\operatorname{Imp})\right] \overrightarrow{\mathbf{z}} \\
& =\overrightarrow{\mathbf{z}}+\frac{1}{\left|\triangle_{\mathbf{q}}\right|} \varphi(\operatorname{Imq}) \tau(\operatorname{Imp}) \overrightarrow{\mathbf{z}} \\
& =\overrightarrow{\mathbf{z}}+\frac{1}{\left\|\mathcal{E}_{\mathbf{q}}\right\|\left\|\mathcal{E}_{\mathbf{p}}\right\|}(\operatorname{Imq}) \overrightarrow{\mathbf{z}}(\operatorname{Imp})
\end{aligned}
$$

If $\mathbf{p} \neq \overline{\mathbf{q}}$ in $\mathbf{q} \mathbf{x}=\mathbf{x p}$, then we write $\mathbf{z}=\operatorname{Imq}$ and $\mathbf{z}=\left\|\mathcal{E}_{\mathbf{q}}\right\|\left\|\mathcal{E}_{\mathbf{p}}\right\|$ in

$$
\mathbf{x}=\mathbf{z}+\frac{1}{\left\|\mathcal{E}_{\mathbf{q}}\right\|\left\|\mathcal{E}_{\mathbf{p}}\right\|}(\operatorname{Im} \mathbf{q}) \overrightarrow{\mathbf{z}}(\operatorname{Im} \mathbf{p})
$$

respectively, it becomes

$$
x_{1}=\operatorname{Im} \mathbf{q}+\operatorname{Imp} \quad \text { and } \quad x_{2}=\left\|\mathcal{E}_{\mathbf{q}}\right\|\left\|\mathcal{E}_{\mathbf{p}}\right\|+\operatorname{Imq} \operatorname{Im} \mathbf{p} .
$$

Thus,

$$
\mathbf{x}=\lambda_{0}[\operatorname{Im} \mathbf{q}+\operatorname{Im} \mathbf{p}]+\lambda_{1}\left[\left\|\mathcal{E}_{\mathbf{q}}\right\|\left\|\mathcal{E}_{\mathbf{p}}\right\|+\operatorname{Imq} \mathbf{I m} \mathbf{p}\right]
$$

is also a solution of $\mathbf{q x}=\mathbf{x p}$ under the conditions $\operatorname{Req}=\operatorname{Rep}$ and $\left\|\mathcal{E}_{\mathbf{q}}\right\|=\left\|\mathcal{E}_{\mathbf{p}}\right\|$. The independence of $x_{1}$ and $x_{2}$ can be seen from two simple facts that $\operatorname{Re} x_{1}=0$ and $\operatorname{Re} x_{1} \neq 0$. Accordingly

$$
\mathbf{x}=\lambda_{0}[\operatorname{Imq}+\operatorname{Imp}]+\lambda_{1}\left[\left\|\mathcal{E}_{\mathbf{q}}\right\|\left\|\mathcal{E}_{\mathbf{p}}\right\|+\operatorname{Imq} \mathbf{I m} \mathbf{p}\right]
$$

is exactly the general solution to $\mathbf{q x}=\mathbf{x p}$, since the rank of $\Omega_{\mathbf{q p}}$ is 2 .

Example 13. Let's take the non-parabolic hybrid numbers $\mathbf{q}=2+\mathbf{i}+\varepsilon+2 \mathbf{h}$ and $\mathbf{p}=2+2 \mathbf{i}+2 \varepsilon+\mathbf{h}$. The linear equation $\mathbf{q x}=\mathbf{x p}$ has a nonzero solution. So, we have $\operatorname{Im} \mathbf{q}+\operatorname{Im} \mathbf{p}=3 \mathbf{i}+3 \varepsilon+3 \mathbf{h}$ and $\left\|\mathcal{E}_{\mathbf{q}}\right\|\left\|\mathcal{E}_{\mathbf{p}}\right\|+\operatorname{Imq} \operatorname{Im} \mathbf{p}=$ $9-3 \mathbf{i}$ and the general solution is $\mathbf{x}=\lambda_{0}[3 \mathbf{i}+3 \varepsilon+3 \mathbf{h}]+\lambda_{1}[9-3 \mathbf{i}]$.

Theorem 14. Let $\mathbf{q}$ and $\mathbf{p} \in \mathbb{K}$ be two parabolic hybrid numbers. Then we have the following properties. 1. The linear equation $\mathbf{q x}=\mathbf{x p}$ has a nonzero solution. Also, $\mathbf{q}$ and $\mathbf{p}$ are similar if and only if $\operatorname{Req}=\operatorname{Re} \mathbf{p}$, and $\left\|\mathcal{E}_{\mathbf{q}}\right\|=\left\|\mathcal{E}_{\mathbf{p}}\right\|=0$. 2. The general solution of $\mathbf{q} \mathbf{x}=\mathbf{x p}$ is

$$
\mathbf{x}=\left(\frac{z_{4} p_{3}+z_{4} q_{3}}{p_{2}-p_{3}-q_{2}+q_{3}}-\frac{z_{3} p_{4}+z_{3} q_{4}}{p_{2}-p_{3}-q_{2}+q_{3}}\right)+\left(\frac{z_{3} p_{2}-z_{3} q_{2}}{p_{2}-p_{3}-q_{2}+q_{3}}+\frac{z_{4} p_{4}-z_{4} q_{4}}{p_{2}-p_{3}-q_{2}+q_{3}}\right) \mathbf{i}+z_{3} \varepsilon+z_{4} \mathbf{h}
$$

where $\mathbf{z}=z_{1}+z_{2} \mathbf{i}+z_{3} \varepsilon+z_{4} \mathbf{h} \in \mathbb{K}$ is an arbitrary and $p_{2} \neq q_{2}, p_{2}-p_{3} \neq q_{2}-q_{3}$. 
Proof. Matrix representation of the equation $\mathbf{q x}=\mathbf{x p}$ is $\Omega_{\mathbf{q p}} \overrightarrow{\mathbf{x}}=\mathbf{c}$. Since $\mathbf{q}$ and $\mathbf{p}$ are similar parabolic hybrid numbers, inverse of the matrix $\Omega_{\mathbf{q p}}$ can be calculated from Theorem 4 . According to the definition of similarty for parabolic hybrid numbers, we have $\mathrm{s}=0$ and the matrix

$$
\begin{gathered}
\Omega_{\mathbf{q p}}= \\
{\left[\begin{array}{cccc}
0 & p_{2}-p_{3}-q_{2}+q_{3} & q_{2}-p_{2} & q_{4}-p_{4} \\
q_{2}-p_{2} & -p_{4}-q_{4} & 0 & p_{2}+q_{2} \\
q_{3}-p_{3} & -p_{4}-q_{4} & p_{4}+q_{4} & p_{2}-p_{3}+q_{2}-q_{3} \\
q_{4}-p_{4} & p_{3}+q_{3} & -p_{2}-q_{2} & 0
\end{array}\right] .}
\end{gathered}
$$

We can easily see that the condition $C \mathbf{R}^{-1} \mathrm{~B}=\mathrm{D}$ of Theorem 4 is satisfied where

$$
\mathrm{C}=
$$

$\left[\begin{array}{cc}q_{3}-p_{3} & -p_{4}-q_{4} \\ q_{4}-p_{4} & p_{3}+q_{3}\end{array}\right], \mathrm{B}=$

$\left[\begin{array}{cc}q_{2}-p_{2} & q_{4}-p_{4} \\ 0 & p_{2}+q_{2}\end{array}\right]$

$\mathrm{D}=[$

$$
\begin{aligned}
& \begin{array}{ccc}
p_{4}+q_{4} & p_{2}-p_{3}+q_{2}-q_{3} & \text { and } \mathbf{R}= \\
-p_{2}-q_{2} & 0 &
\end{array} \\
& {\left[\begin{array}{cc}
0 & p_{2}-p_{3}-q_{2}+q_{3} \\
q_{2}-p_{2} & -p_{4}-q_{4}
\end{array}\right]}
\end{aligned}
$$

Therefore, the generalized inverse of the matrix $\Omega_{\mathrm{qp}}$ is

$$
\Omega_{\mathrm{qp}}^{-1}=
$$

$$
\left[\begin{array}{cccc}
-\frac{p_{4}+q_{4}}{\left(p_{2}-q_{2}\right)\left(p_{2}-p_{3}-q_{2}+q_{3}\right)} & -\frac{1}{p_{2}-q_{2}} & 0 & 0 \\
\frac{p_{2}-p_{3}-q_{2}+q_{3}}{0} & 0 & 0 & 0 \\
0 & 0 & 0 & 0 \\
0 & 0 & 0 & 0
\end{array}\right] .
$$

One can see that this matrix satisfies the condition of the definition 3 which is $\Omega_{\mathbf{q p}} \Omega_{\mathbf{q p}}^{-1} \Omega_{\mathbf{q p}}=\Omega_{\mathrm{qp}}$. Thus, we obtain the general solution of $\mathbf{q} \mathbf{x}=\mathbf{x p}$ as follows, according to the Theorem 5

$$
\mathbf{x}=
$$

$$
\left[\begin{array}{c}
z_{4} \frac{p_{3}+q_{3}}{p_{2}-p_{3}-q_{2}+q_{3}}-z_{3} \frac{p_{4}+q_{4}}{p_{2}-p_{3}-q_{2}+q_{3}} \\
z_{3} \frac{p_{2}-q_{2}}{p_{2}-p_{3}-q_{2}+q_{3}}+z_{4} \frac{p_{4}-q_{4}}{p_{2}-p_{3}-q_{2}+q_{3}} \\
z_{3} \\
z_{4}
\end{array}\right] .
$$


Example 15. Let's take the similar parabolic hybrid numbers, $\mathbf{q}=3-2 \mathbf{i}+3 \varepsilon+4 \mathbf{h}, \mathbf{p}=3-8 \mathbf{i}+5 \varepsilon-12 \mathbf{h}$, the linear equation $\mathbf{q} \mathbf{x}=\mathbf{x p}$ has a general solution

$$
\mathbf{x}=-\left(z_{4}+z_{3}\right)+\left(z_{3} \frac{3}{4}+2 z_{4}\right) \mathbf{i}+z_{3} \varepsilon+z_{4} \mathbf{h}
$$

where $z=z_{1}+z_{2} \mathbf{i}+z_{3} \varepsilon+z_{4} \mathbf{h}$ is an arbitrary hybrid number. This linear equation can be written as

$$
\Omega_{\mathrm{qp}} \overrightarrow{\mathrm{x}}=[
$$

$\begin{array}{cccc}0 & -8 & 6 & 16 \\ 6 & 8 & 0 & -10 \\ -2 & 8 & -8 & -18 \\ 16 & 8 & 10 & 0\end{array} \quad \overrightarrow{\mathrm{x}}=0$. We have $\operatorname{det} \Omega_{\mathbf{q p}}=0$ and this matrix provide generalized inverse condition,

$$
\mathrm{CR}^{-1} \mathrm{~B}=[
$$

$-28$

168

$\begin{array}{ccc}\frac{1}{6} & \frac{1}{6} \\ -\frac{1}{8} & 0\end{array}$

$\begin{array}{cc}6 & 16 \\ 0 & -10\end{array}=[$

$\begin{array}{cc}-8 & -18 \\ 10 & 0\end{array}=$ D. Therefore, the generalized inverse of $\Omega_{\mathbf{q p}}$ is

$$
\Omega_{\mathbf{q p}}^{-1}=[
$$

$\begin{array}{cccc}\frac{1}{6} & \frac{1}{6} & 0 & 0 \\ -\frac{1}{8} & 0 & 0 & 0\end{array}$

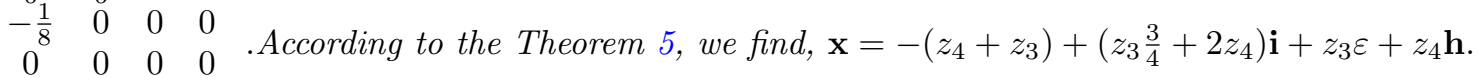

$\begin{array}{llll}0 & 0 & 0 & 0\end{array}$

Now, let's give a direct conclusion of Theorem 12.

Corollary 16. The equation $\mathbf{q x}=\mathbf{x} \overline{\mathbf{q}}$ in hybrid numbers always has a nonzero solution. Moreover, $\mathbf{q} \sim \overline{\mathbf{q}}=$ $q_{1}+\left\|\mathcal{E}_{\mathbf{q}}\right\| \mathbf{V}$ where $\mathbf{V}=\{\mathbf{i}, \varepsilon, \mathbf{h}\}$.

Therefore, we have the following tables:

\begin{tabular}{|c|c|c|c|}
\hline Type $\backslash$ Character & Spacelike & Lightlike & Timelike \\
\hline Hyperbolic & $q_{1}+\left\|\mathcal{E}_{\mathbf{q}}\right\|$ & $q_{1}$ & $q_{1}+\left\|\mathcal{E}_{\mathbf{q}}\right\|$ \\
\hline Parabolic & - & 0 & $q_{1}$ \\
\hline Elliptic & - & - & $q_{1}+\left\|\mathcal{E}_{\mathbf{q}}\right\| \mathbf{i}$ \\
\hline
\end{tabular}

Table 3 : Similarity of $\mathbf{q}$ in $\mathbb{K}$ 


\begin{tabular}{|c|c|c|c|}
\hline Type $\backslash$ Character & Spacelike & Lightlike & Timelike \\
\hline Hyperbolic & $\begin{array}{c}\lambda_{0}\left(\left\|\mathcal{E}_{\mathbf{q}}\right\| \mathbf{h}+\operatorname{Imq}\right) \\
+\lambda_{1}\left(\left\|\mathcal{E}_{\mathbf{q}}\right\|+(\operatorname{Imq}) \mathbf{h}\right)\end{array}$ & $\lambda_{0} \operatorname{Imq}$ & $\begin{array}{c}\lambda_{0}\left(\left\|\mathcal{E}_{\mathbf{q}}\right\| \mathbf{h}+\operatorname{Imq}\right) \\
+\lambda_{1}\left(\left\|\mathcal{E}_{\mathbf{q}}\right\|+(\operatorname{Imq}) \mathbf{h}\right)\end{array}$ \\
\hline Parabolic & - & 0 & $\lambda_{0} \operatorname{Imq}$ \\
\hline Elliptic & - & - & $\begin{array}{c}\left.\lambda_{0}\left(\left\|\mathcal{E}_{\mathbf{q}}\right\| \mathbf{i}+\operatorname{Imq}\right)\right) \\
+\lambda_{1}\left(\left\|\mathcal{E}_{\mathbf{q}}\right\|+(\operatorname{Imq}) \mathbf{i}\right)\end{array}$ \\
\hline
\end{tabular}

Table 5: General solution of $\mathbf{q x}=\mathbf{x} \widehat{\mathbf{q}}$ in $\mathbb{K}$

where $\lambda_{0}, \lambda_{1}$ are arbitrary real numbers..

Theorem 17. Let $\mathbf{q}$ and $\mathbf{p}$ are non-parabolic similar hybrid numbers. Then, the equation $\mathbf{q} \mathbf{x}-\mathbf{x p}=\mathbf{c}$ has a solution if and only if $\mathbf{q} \mathbf{c}=\mathbf{c} \overline{\mathbf{p}}$. Moreover, the general solution of $\mathbf{q x}-\mathbf{x p}=\mathbf{c}$ can be expressed as

$$
\mathbf{x}=\frac{1}{4\left|\triangle_{\mathbf{q}}\right|}(\mathbf{q} \mathbf{c}-\mathbf{c p})+\mathbf{z}+\frac{1}{\left|\triangle_{\mathbf{q}}\right|}(\operatorname{Im} \mathbf{q}) \mathbf{z}(\operatorname{Im} \mathbf{p})
$$

where $\mathbf{z}$ is an arbitrary hybrid number.

Proof. The equation $\mathbf{q} \mathbf{x}-\mathbf{x p}=\mathbf{c}$ can be written as,

$$
[\varphi(\mathbf{q})-\tau(\mathbf{p})] \overrightarrow{\mathbf{x}}=\Omega_{\mathbf{q p}} \overrightarrow{\mathbf{x}}=\overrightarrow{\mathbf{c}} .
$$

This equation is solvable if only if $\Omega_{\mathbf{q p}} \Omega_{\mathbf{q p}}^{-1} \overrightarrow{\mathbf{c}}=\overrightarrow{\mathbf{c}}$ which is equivalent to

$$
[\varphi(\operatorname{Imq}) \tau(\operatorname{Imp})] \overrightarrow{\mathbf{c}}=-\left|\triangle_{\mathbf{q}}\right| \overrightarrow{\mathbf{c}} .
$$

Returning it from matrix form to hybridian form, we can write the equation

$$
(\operatorname{Imq}) \mathbf{c}(\operatorname{Imp})=-\left|\triangle_{\mathbf{q}}\right| \overrightarrow{\mathbf{c}}
$$

Therefore, we find

$$
\begin{aligned}
(\operatorname{Im} \mathbf{q})(\operatorname{Imq}) \mathbf{c}(\operatorname{Imp}) & =-(\operatorname{Imq})\left|\triangle_{\mathbf{q}}\right| \overrightarrow{\mathbf{c}} \\
\overrightarrow{\mathbf{c}}(\operatorname{Imp}) & =-(\operatorname{Imq}) \overrightarrow{\mathbf{c}}
\end{aligned}
$$

and then $\mathbf{q} \mathbf{c}=\mathbf{c} \overline{\mathbf{p}}$. In that case, the general solution of () can be given as

$$
\mathbf{x}=\Omega_{\mathbf{q p}}^{-1} \overrightarrow{\mathbf{c}}+2\left[I_{4}+\frac{1}{\left|\triangle_{\mathbf{q}}\right|}(\operatorname{Im} \mathbf{q})(\operatorname{Imp})\right] \overrightarrow{\mathbf{z}},
$$

where $\overrightarrow{\mathbf{z}}=(a, b, c, d)$ is an arbitrary real vector. Thus, we can find

$$
\mathbf{x}=\frac{1}{4\left|\triangle_{\mathbf{q}}\right|}(\mathbf{q} \mathbf{c}-\mathbf{c p})+\mathbf{z}+\frac{1}{\left|\triangle_{\mathbf{q}}\right|}(\operatorname{Imq}) \mathbf{z}(\operatorname{Imp}) .
$$


Example 18. Let's find take the general solution of equation $\mathbf{q x}-\mathbf{x p}=\mathbf{c}$ for the non-parabolic hybrid numbers $\mathbf{q}=2+\mathbf{i}+2 \varepsilon+3 \mathbf{h}, \mathbf{p}=2+2 \mathbf{i}+\mathbf{3} \varepsilon+\mathbf{2 h}, \mathbf{c}=\mathbf{i}+\varepsilon-\mathbf{h}$. We have,

$$
(\mathbf{q} \mathbf{c}-\mathbf{c p})=\varphi(\mathbf{q}) \mathbf{c}-\tau(\mathbf{p}) \mathbf{c}=
$$

$\left[\begin{array}{llll}-2 & -8 & 2 & 2\end{array}\right]^{\mathrm{t}}$. Therefore, according to the Theorem 17, we obtain the the general solution as

$$
\mathbf{x}=\frac{1}{24}
$$

$\left[\begin{array}{c}46 a-10 b+8 c-2 d-1 \\ 8 b-8 a+8 c+16 d-4 \\ 2 a+10 b+16 c+26 d+1 \\ 2 a+10 b+16 c+26 d+1\end{array}\right]$

where $\overrightarrow{\mathbf{z}}=(a, b, c, d)$ is an arbitrary real vector.

Theorem 19. Let $\mathbf{q}=q_{1}+q_{2} \mathbf{i}+q_{3} \varepsilon+q_{4} \mathbf{h}$ and $\mathbf{p}=p_{1}+p_{2} \mathbf{i}+p_{3} \varepsilon+p_{4} \mathbf{h}$ be parabolic similar hybrid numbers with $p_{2} \neq q_{2}, p_{2}-p_{3} \neq q_{2}-q_{3}$. Then, the equation

$$
\mathbf{q x}-\mathbf{x p}=\mathbf{c}
$$

has a solution where

$$
\mathbf{c}=c_{1}+c_{2} \mathbf{i}+\left(\frac{-c_{1}\left(p_{4}+q_{4}\right)}{p_{2}-q_{2}}-\frac{c_{2}\left(q_{3}-p_{3}\right)}{p_{2}-q_{2}}\right) \varepsilon+\left(\frac{c_{1}\left(p_{2}+q_{2}\right)}{p_{2}-q_{2}}+\frac{c_{2}\left(p_{4}-q_{4}\right)}{p_{2}-q_{2}}\right) \mathbf{h} \in \mathbb{K} .
$$

The general solution of $\mathbf{q} \mathbf{x}-x \mathbf{p}=\mathbf{c}$ can be expressed as

$$
\mathbf{x}=x_{1}+x_{2} \mathbf{i}+z_{3} \varepsilon+z_{4} \mathbf{h}
$$

where

$$
\begin{aligned}
& x_{1}=\frac{z_{4} p_{3}+z_{4} q_{3}}{p_{2}-p_{3}-q_{2}+q_{3}}-\frac{c_{1} p_{4}+c_{1} q_{4}}{\left(p_{2}-q_{2}\right)\left(p_{2}-p_{3}-q_{2}+q_{3}\right)}-\frac{c_{2}}{p_{2}-q_{2}}-\frac{z_{3} p_{4}+z_{3} q_{4}}{p_{2}-p_{3}-q_{2}+q_{3}} \\
& x_{2}=\frac{c_{1}}{p_{2}-p_{3}-q_{2}+q_{3}}+\frac{z_{3} p_{2}-z_{3} q_{2}}{p_{2}-p_{3}-q_{2}+q_{3}}+\frac{z_{4} p_{4}-z_{4} q_{4}}{p_{2}-p_{3}-q_{2}+q_{3}} .
\end{aligned}
$$

and $\mathbf{z}=z_{1}+z_{2} \mathbf{i}+z_{3} \varepsilon+z_{4} \mathbf{h}$ is an arbitrary hybrid number.

Proof. Since $\mathbf{q}$ and $\mathbf{p}$ are similar parabolic hybrid numbers, the generalized inverse of $\Omega_{\mathbf{q p}}$ is as given in the Theorem 4. So, its general solution is

$$
\begin{aligned}
& \mathrm{x}= \\
& {\left[\begin{array}{c}
z_{4} \frac{p_{3}+q_{3}}{p_{2}-p_{3}-q_{2}+q_{3}}-c_{1} \frac{p_{4}+q_{4}}{\left(p_{2}-q_{2}\right)\left(p_{2}-p_{3}-q_{2}+q_{3}\right)}-\frac{c_{2}}{p_{2}-q_{2}}-z_{3} \frac{p_{4}+q_{4}}{p_{2}-p_{3}-q_{2}+q_{3}} \\
\frac{c_{1}}{p_{2}-p_{3}-q_{2}+q_{3}}+z_{3} \frac{p_{2}-q_{2}}{p_{2}-p_{3}-q_{2}+q_{3}}+z_{4} \frac{p_{4}-q_{4}}{p_{2}-p_{3}-q_{2}+q_{3}} \\
z_{3} \\
z_{4}
\end{array}\right]}
\end{aligned}
$$


Example 20. Let's find the general solution of equation $\mathbf{q x}-\mathbf{p x}=\mathbf{c}$ for the parabolic hybrid numbers $\mathbf{q}=-2-4 \mathbf{i}+6 \varepsilon+8 \mathbf{h}, \mathbf{p}=-2-\mathbf{i}+\mathbf{1 2} \varepsilon+\mathbf{5 h}, c=1+\mathbf{i}+\mathbf{2 9} \varepsilon-4 \mathbf{h}$. According to Theorem 19, the solution of $\Omega_{\mathrm{qp}} \mathbf{x}=\mathbf{c}$ is

$$
\mathbf{x}=
$$

$\left[\frac{28}{15}-\frac{32}{15} z_{4}-\frac{13}{15} z_{3} \quad \frac{1}{15} z_{4}-\frac{1}{15} z_{3}+\frac{1}{15} \quad z_{3} \quad z_{4}\right]{ }^{\mathrm{t}}$ where $\mathbf{z}=z_{1}+z_{2} \mathbf{i}+z_{3} \varepsilon+z_{4} \mathbf{h}$ is an arbitrary hybrid number.

Theorem 21. Let $\mathbf{q}$ and $\mathbf{p}$ be two non-similar hybrid numbers. Namely, Req $\neq \operatorname{Rep}$ or $\left\|\mathcal{E}_{\mathbf{q}}\right\| \neq\left\|\mathcal{E}_{\mathbf{p}}\right\|$. Then the equation $\mathbf{q} \mathbf{x}-\mathbf{x p}=\mathbf{c}$ has a unique solution

$$
\begin{aligned}
x & =\left(2 \mathrm{sq}+\|\mathbf{p}\|^{2}-\|\mathbf{q}\|^{2}\right)^{-1}(\mathbf{q} \mathbf{c}-\mathbf{c} \overline{\mathbf{p}}) \\
& =(\mathbf{q} \mathbf{c}-\mathbf{c} \overline{\mathbf{p}})\left(2 \mathrm{~s} \mathbf{q}+\|\mathbf{p}\|^{2}-\|\mathbf{q}\|^{2}\right)^{-1}
\end{aligned}
$$

where $\mathrm{s}=\operatorname{Req}-$ Rep.

Proof. Since $\mathbf{q}$ and $\mathbf{p}$ are not similar, $\operatorname{det} \Omega_{\mathbf{q p}} \neq 0$ and $\Omega_{\mathbf{q p}}$ has an inverse which it can be expressed as Theorem 8-3

$$
\begin{aligned}
& \Omega_{\mathbf{q p}}^{-1}=\varphi^{-1}\left(2 \mathbf{s q}+\|\mathbf{p}\|^{2}-\|\mathbf{q}\|^{2}\right)[\varphi(\mathbf{q})-\tau(\overline{\mathbf{p}})] \\
& \Omega_{\mathbf{q p}}^{-1}=\tau^{-1}\left(-2 \mathbf{s q}+\|\mathbf{p}\|^{2}-\|\mathbf{q}\|^{2}\right)[\varphi(\overline{\mathbf{q}})-\tau(\mathbf{p})]
\end{aligned}
$$

Thus, we get

$$
\begin{aligned}
\overrightarrow{\mathbf{x}} & =\Omega_{\mathbf{q p}}^{-1} \overrightarrow{\mathbf{c}} \\
& =\varphi^{-1}\left(2 \mathrm{sq}+\|\mathbf{p}\|^{2}-\|\mathbf{q}\|^{2}\right)[\varphi(\mathbf{q})-\tau(\overline{\mathbf{p}})] \overrightarrow{\mathbf{c}} \\
& =\left(2 \mathbf{s q}+\|\mathbf{p}\|^{2}-\|\mathbf{q}\|^{2}\right)^{-1}(\mathbf{q} \mathbf{c}-\mathbf{c} \overline{\mathbf{p}})
\end{aligned}
$$

and

$$
\begin{aligned}
\overrightarrow{\mathbf{x}} & =\Omega_{\mathbf{q p}}^{-1 \vec{c}} \\
& =\tau^{-1}\left(-2 \mathbf{s} \mathbf{q}+\|\mathbf{p}\|^{2}-\|\mathbf{q}\|^{2}\right)[\varphi(\overline{\mathbf{q}})-\tau(\mathbf{p})] \overrightarrow{\mathbf{c}} \\
& =(\mathbf{q} \mathbf{c}-\mathbf{c} \overline{\mathbf{p}})\left(2 \mathbf{s q}+\|\mathbf{p}\|^{2}-\|\mathbf{q}\|^{2}\right)^{-1} .
\end{aligned}
$$

Example 22. Let's find the unique solution of the equation $\mathbf{q x}-x \mathbf{p}=\mathbf{c}$ for the hybrid numbers, $\mathbf{q}=2+\mathbf{i}+$ $\varepsilon+2 \mathbf{h}, \mathbf{p}=1+2 \mathbf{i}+2 \varepsilon+\mathbf{h}, \mathbf{c}=-\mathbf{2}+3 \mathbf{i}+\varepsilon+5 \mathbf{h}$. Since $\mathbf{q}$ and $\mathbf{p}$ are not similar, we have

$$
\left(2 \mathbf{s q}+\|\mathbf{p}\|^{2}-\|\mathbf{q}\|^{2}\right)=2(2+\mathbf{i}+\varepsilon+2 \mathbf{h})-4-(-1)=1+2 \mathbf{i}+2 \varepsilon+4 \mathbf{h}
$$

and 


$$
\mathbf{q} \mathbf{c}-\mathbf{c} \overline{\mathbf{p}}=\varphi(\mathbf{q}) \mathbf{c}-\tau(\overline{\mathbf{p}}) \mathbf{c}=(16,-11,-7,-3)^{\mathrm{t}}
$$

Therefore, we obtain

$$
\begin{aligned}
& {\left[\begin{array}{llll}
16 & -11 & -7 & -3
\end{array}\right]^{\mathrm{t}}} \\
& {\left[\begin{array}{llll}
-\frac{42}{19} & \frac{81}{19} & \frac{55}{19} & \frac{59}{19}
\end{array}\right] \mathrm{t}} \\
& =-\frac{42}{19}+\frac{81}{19} \mathbf{i}+\frac{55}{19} \varepsilon+\frac{59}{19} \mathbf{h} \text {. }
\end{aligned}
$$

\section{Conclusions}

In this article, we examined the similarity of hybrid numbers and linear equations with hybrid coefficients. For this purpose, we have examined the appropriate studies in quaternions. We found similarities in our examination. As a result, when we examine and categorize a hybrid number according to its module and casual character, we obtained different results in terms of the similarity of the two hybrid numbers. However, while solving linear equations with hybrid number coefficients, in the solution of similar parabolic hybrid equations naturally emerged different from the quaternions. This is because the $\left\|\mathcal{E}_{\mathbf{q}}\right\|$ of a parabolic hybrid number $\mathbf{q}$ is zero. This difficulty is solved by the generalized inverse theorem which is used to find the inverse of non-square matrices. The examination of these situations has brought a new solution to the systems of linear equations.

\section{References}

